International Mathematical Forum, 2, 2007, no. 5, 237 - 241

\title{
The structure of module amenable Banach algebras
}

\author{
S. A. Hosseiniun and D. Ebrahimi Bagha \\ Department of Mathematics, Shahid Beheshti University \\ Evin, Teheran, Iran \\ r-hosseini@cc.sbu.ac.ir \\ D-Ebrahimi@cc.sbu.ac.ir
}

\begin{abstract}
In this paper, we study the module amenability of Banach algebras and characterize it in terms the concepts spliting and admissiblity of short exact sequences.
\end{abstract}

Mathematics Subject Classification: 43A07, 46H25

Keywords: Amenability, Module Amenability, Spliting admissiblity

\section{MODULE AMENABILITY}

All of the definitions in the following are in $[\mathrm{A}]$.

Let $\mathfrak{A}$ and $A$ be Banach algebras such that $A$ is a Banach $\mathfrak{A}$-bimodule with compatible actions, that is

$$
\alpha \cdot(a b)=(\alpha \cdot a) b, a(\alpha \cdot b)=(a \cdot \alpha) b \quad(a, b \in A, \alpha \in \mathfrak{A})
$$

Let $X$ be a Banach A-bimodule and a Banach $\mathfrak{A}$-bimodule with compatible actions, that is

$\alpha \cdot(a \cdot x)=(\alpha \cdot a) \cdot x,(a \cdot \alpha) \cdot x=a \cdot(\alpha \cdot x),(\alpha \cdot x) \cdot a=\alpha \cdot(x \cdot a) \quad(a \in A, \alpha \in \mathfrak{A}, x \in X)$ and the same for the right or two-sided actions. Then we say that $X$ is a Banach $\mathrm{A}-\mathfrak{A}-$ module. If morever

$$
\alpha \cdot x=x \cdot \alpha \quad(\alpha \in \mathfrak{A}, x \in X),
$$

Then $X$ is called a commutative $\mathrm{A}-\mathfrak{A}$-module .

A bounded map $D: A \longrightarrow X$ is called a module derivation if

$$
D(a \pm b)=D(a) \pm D(b), \quad D(a b)=(D a) \cdot b+a \cdot(D b) \quad(a, b \in A)
$$

and

$$
D(\alpha \cdot a)=\alpha \cdot D(a), D(a \cdot \alpha)=D(a) \cdot \alpha \quad(\alpha \in \mathfrak{A}, a \in A) .
$$


Note that $D: A \longrightarrow X$ is bounded if there exist $M>0$ such that $\|D(a)\| \leq$ $M\|a\|$, for each $a \in A$. Although $D$ is not necessarily linear ,but still its boundedness implies its norm continuity. When $X$ is commutative, each $x \in X$ defines a module derivation

$$
D_{x}(a)=a \cdot x-x \cdot a \quad(a \in A) .
$$

These are called inner module derivations.

Definition 2.1. $A$ is called module amenable (as an $\mathfrak{A}$-module) if for any commutative Banach $\mathrm{A}-\mathfrak{A}$-module $X$, each module derivation $D: A \longrightarrow X^{*}$ is inner.

Next let $A \widehat{\otimes}_{\mathfrak{A}} A$ be the projective module tensor product of $A$ and $A[R]$. This is the quotient of the usual projective tensor product $A \widehat{\otimes} A$ by the closed ideal $I$ generated by elements of the form $\alpha \cdot a \otimes b-a \otimes b \cdot \alpha$ for $\alpha \in \mathfrak{A}, a, b \in A$. We have

$$
\left(A \widehat{\otimes}_{\mathfrak{A}} A\right)^{*} \cong L_{\mathfrak{A}}\left(A, A^{*}\right),
$$

where the right hand side is the space of all $\mathfrak{A}$-module morphisms from $A$ to $A^{*}[R]$. In particular $A \widehat{\otimes}_{\mathfrak{A}} A$ is a Banach $\mathrm{A}-\mathfrak{A}-$ module consider $w \in$ $L(A \widehat{\otimes} A, A)$ define by $w(a \otimes b)=a b(a, b \in A)$ and extended by linearity

Then both $w$ and its second conjugate $w^{* *} \in L\left((A \widehat{\otimes} A)^{* *}, A^{* *}\right)$ are $\mathrm{A}-\mathfrak{A}-$ module homomorphisms. Let $J$ be the closed ideal of $A$ generated by $w(I)$. We define $\tilde{w}: A \hat{\otimes}_{\mathfrak{A}} A=A \hat{\otimes} A / I \longrightarrow \frac{A}{J}$ by

$$
\tilde{w}(a \otimes b+I)=a b+J \quad(a, b \in A) .
$$

This extends to an element $\tilde{w} \in L\left(A \hat{\otimes}_{\mathfrak{A}} A, \frac{A}{J}\right)$ and both $\tilde{w}$ and its dual conjugate $\tilde{w}^{* *} \in L\left(\left(A \hat{\otimes}_{\mathfrak{A}} A\right)^{* *}, \frac{A^{* *}}{J^{\perp \perp}}\right)$ are A-A-module homomorphisms.

Also $\frac{A}{J}$ and $A \hat{\otimes}_{\mathfrak{A}} A$ are Banach $\frac{A}{J}-\mathfrak{A}-$ module and $\tilde{w}$ and $\tilde{w}^{* *}$ are $\frac{A}{J}-$ $\mathfrak{A}$-module homomorphisms.

Definition 2.2. A bounded net $\left\{e_{\alpha}\right\}$ in $A \widehat{\otimes}_{\mathfrak{A}} A$ is called a module approximate diagonal for $A$ if for each $a \in A$,

$$
\lim _{\alpha}\left[e_{\alpha} \cdot(a+J)-(a+J) \cdot e_{\alpha}\right]=0, \lim _{\alpha} \tilde{w}\left(e_{\alpha}\right)(a+J)=a+J .
$$

An element $\tilde{M} \in\left(A \widehat{\otimes}_{\mathfrak{A}} A\right)^{* *}$ is called a module virtual diagonal for $A$ if for each $a \in A$,

$$
\left(\tilde{w}^{* *} \tilde{M}\right) \cdot(a+J)=a+J, \quad(a+J) \cdot \tilde{M}=\tilde{M} \cdot(a+J) .
$$


If $\left\{e_{\alpha}\right\}$ be a module approximate diagonal for $A$ then $\left\{\tilde{w}\left(e_{\alpha}\right)\right\}$ is a bounded approximate identity for $\frac{A}{J}$.

The following results are proved as in the classical case $[A]$.

Theorem 2.3. The following are equivalent:

(i) $A$ is module amenable and $\frac{A}{J}$ has a bounded approximate identity .

(ii) A has a module approximate diagonal.

(iii) $A$ has a module virtual diagonal .

Proposition 2.4. Let $\frac{A}{J}$ be a commutative Banach $\mathfrak{A}$-module. if $A$ is module amenable then $\frac{A}{J}$ has a bounded approximate identity.

\section{CHARACTERIZATION OF MODULE AMENABILITY}

If $X, Y$ and $Z$ are Banach $\frac{A}{J}-\mathfrak{A}-$ modules and $f: X \longrightarrow Y, g: Y \longrightarrow Z$ are $\frac{A}{J}-\mathfrak{A}-$ module homomorphisms, Then the sequence

$$
\Sigma: 0 \longrightarrow X \longrightarrow Y \longrightarrow Z \longrightarrow 0
$$

is exact if $f$ is one -to-one , $\operatorname{Im} g=Z$, and $\operatorname{Im} f=k e r g$. The exact sequence $\Sigma$ is admissible if there is a $\mathfrak{A}$-module homomorphism $F: Y \longrightarrow X$ such $F f=I$ on $X$. The exact sequence $\Sigma$ splits if there is a $\frac{A}{J}-\mathfrak{A}-$ modules homomorphism $F: Y \longrightarrow X$ such that $F f=I$ on $X$.

If $K$ is the kernel of $\tilde{w}$, and $\frac{A}{J}$ has a bounded approximate identity, then the sequence

$$
\tilde{\Pi}: 0 \longrightarrow K \stackrel{i}{\longrightarrow} A \widehat{\otimes}_{\mathfrak{A}} A \stackrel{\tilde{\omega}}{\longrightarrow} \frac{A}{J} \longrightarrow 0
$$

is exact as a sequence of Banach $\frac{A}{J}$ - $A$-modules, and the same is true for the dual sequence

$$
\tilde{\Pi}^{*}: 0 \longrightarrow\left(\frac{A}{J}\right)^{*} \stackrel{\tilde{\omega}^{*}}{\longrightarrow}\left(A \widehat{\otimes}_{\mathfrak{A}} A\right)^{*} \stackrel{i^{*}}{\longrightarrow} K^{*} \longrightarrow 0
$$

Proposition 3.1. Let $\Sigma: 0 \longrightarrow X \stackrel{f}{\longrightarrow} Y \stackrel{g}{\longrightarrow} Z \longrightarrow 0$ be a short exact sequence of Banach $\frac{A}{J}-\mathfrak{A}$-modules. If there exists a $\mathfrak{A}-$ module morphism $F: Y \longrightarrow X$ satisfying $F f=I$ on $X$ then there exists a unique $\mathfrak{A}$-module morphism $G: Z \longrightarrow Y$ satisfying $g G=I$ on $Y$ and conversely. moreover $F$ is 
an $\frac{A}{J}-\mathfrak{A}$-module morphism if and only if $G$ is.

Proof. is similar to [1].

Lemma 3.2. Let $\frac{A}{J}$ and $A \widehat{\otimes}_{\mathfrak{A}} A$ are commutative Banach $\mathfrak{A}$-modules. If $\frac{A}{J}$ has an identity, the sequence $\tilde{\Pi}, \tilde{\Pi}^{*}$ are admissible . If $\frac{A}{J}$ has a bounded approximate identity , $\tilde{\Pi}^{*}$ is admissible.

Proof. Let $\frac{A}{J}$ has an identity $e+J, \theta(a+J)=a \otimes e+I$ is a $\mathfrak{A}$-module morphism and a right inverse for $\tilde{w}$, and $\theta^{*}$ is the required left inverse for $\tilde{w}^{*}$ as $\tilde{w} \theta=I$ implies that $\theta^{*} \tilde{w}^{*}=I^{*}$ on $\left(\frac{A}{J}\right)^{*}$. If $\frac{A}{J}$ has a bounded approximate identity $\left\{e_{\alpha}+J\right\}$, let $u \in\left(A \widehat{\otimes}_{\mathfrak{A}} A\right)^{* *}$ be a weak* limit point of $\left\{e_{\alpha} \otimes e_{\alpha}+I\right\}$. Define $\sigma:\left(A \widehat{\otimes}_{\mathfrak{A}} A\right)^{*} \rightarrow\left(\frac{A}{J}\right)^{*}$ by

$$
\langle a+J, \sigma(g)\rangle=\langle(a+J) \cdot g, u\rangle a \in A, g \in\left(A \widehat{\otimes}_{\mathfrak{A}} A\right)^{*} .
$$

$\sigma$ is a $\mathfrak{A}$-module morphism and a left inverse for $\tilde{w}^{*}$.

Theorem 3.3. Let $\frac{A}{J}$ and $A \widehat{\otimes}_{\mathfrak{A}} A$ are commutative Banach $\mathfrak{A}$-modules . The Banach algebra $A$ is module amenable if and only if

(i) $\frac{A}{J}$ has a bounded approximate identity, and

(ii) The exact sequence $\tilde{\Pi}^{*}$ splits.

Proof. If $A$ is module amenable, then $\frac{A}{J}$ has a bounded approximate identity . Let $\tilde{M}$ be a module virtual diagonal for $A$. For $f \in\left(A \widehat{\otimes}_{\mathfrak{A}} A\right)^{*}, a \in A$ define $\langle\theta(f), a+J\rangle=\langle\tilde{M}, f \cdot(a+J)\rangle \cdot \theta$ is a $\frac{A}{J}-\mathfrak{A}$-module morphism from $\left(A \widehat{\otimes}_{\mathfrak{A}} A\right)^{*}$ in to $\left(\frac{A}{J}\right)^{*}$ and $\theta 0 \tilde{w}^{*}=I$ on $\left(\frac{A}{J}\right)^{*}$. Conversely, Suppose that $\frac{A}{J}$ has a bounded approximate identity $\left\{e_{\alpha}+J\right\}$ and $\theta$ is an $\frac{A}{J}-\mathfrak{A}$-module morphism with $\theta \tilde{w}^{*}=I$, on $\left(\frac{A}{J}\right)^{*}$. Suppose that $\left\{e_{\alpha} \otimes e_{\alpha}+I\right\}$ converges weak* to $u \in\left(A \widehat{\otimes}_{\mathfrak{A}} A\right)^{* *}$. Set $\tilde{M}=\theta^{*} \tilde{w}^{* *} \mathfrak{A}$. Then $\tilde{M}$ is a module virtual diagonal for $A$. 
Theorem 3.4. Let $A$ be a module amenable Banach algebra, and let $\Sigma: 0 \longrightarrow X^{*} \longrightarrow Y \longrightarrow Z \longrightarrow 0$ be an admissible short exact sequence of commutative Banach $\frac{A}{J}-\mathfrak{A}-$ module with $X^{*}$ a dual $\frac{A}{J}-\mathfrak{A}$-module. Then $\Sigma$ splits.

Proof. Since $\Sigma$ is admissible, there exists $\tilde{G} \in L_{\mathfrak{A}}(Z, Y)$ satisfying $g \tilde{G}=$ $I$ on $Z$. Define $D(a)=(a+J) \cdot \tilde{G}-\tilde{G}(a+J)$. Then $D$ is a module derivation from $A$ to the bimodule $L_{\mathfrak{A}}(Z, Y)$. Moreover, for $z \in Z g(D a(z))=$ $g[(a+J) \cdot \tilde{G}-\tilde{G} \cdot(a+J)](z)=(a+J) \cdot z-(a+J) \cdot z=0$. Therefore $D(A) \subset L_{\mathfrak{A}}(Z$, ker $g)=L_{\mathfrak{A}}(Z, \operatorname{Im} f)$. Hence $h=f^{-1} D$ is a module derivation from $A$ to the commutative Banach $\mathrm{A}-\mathfrak{A}$-module $L_{\mathfrak{A}}\left(Z, X^{*}\right)=\left(Z \widehat{\otimes}_{\mathfrak{A}} X\right)^{*}$ since $A$ is module amenable, there exists $Q \in L_{\mathfrak{A}}\left(Z, X^{*}\right)$ satisfying $D(a)=$ $(a+J) \cdot \tilde{G}-\tilde{G} \cdot(a+J)=(a+J) \cdot f Q-f Q \cdot(a+J)$. If $G=\tilde{G}-f Q$ then $G \in L_{\mathfrak{A}}(Z, Y)$ and $(a+J) \cdot G=G \cdot(a+J)$ also $G((a+J) \cdot z)=[G \cdot(a+J)](z)=$ $[(a+J) \cdot G](z)=(a+J) \cdot G(z)$ Therefore $G$ is a $\frac{A}{J}$-module morphism and $(g G)(z)=g \tilde{G}(z)-(g f Q)(z)=g \tilde{G}(z)=z$ Therefore $G$ is a right inverse for $g$ , and consequently the sequence $\Sigma$ splits.

Proposition 3.5. Let $A$ be an module amenable, unital Banach algebra . Then any two sided ideal of codimension one such that is a $\mathfrak{A}$-submodule is module amenable.

Proof. Let $M$ be such an ideal and an $\mathfrak{A}$-submodule . for $a \in A$ there exists $\alpha \in \mathfrak{A}, m \in M$ such that $a=m+\alpha 1$. Let $X$ be a commutative Banach M- $\mathfrak{A}-$ module. Now is clearly a commutative A-A-module if we set $a \cdot x=m \cdot x+\alpha x$ , $x \cdot a=x \cdot m+\alpha x(a \in A, x \in X)$. If $D: m \rightarrow X^{\prime}$ is a module derivation, $D$ may be extended as a module derivation from $A$ to $X^{\prime}$ by setting $D(1)=0$ .The module amenability of $A$ then implies the module amenability of $M$.

\section{REFERENCES}

[A] M.Amini, Module amenability for semigroup algebras. Semigroup Forum. 69 (2004), 243254.

[CL] P. C. Curtis, Jr. and R. J. Loy, The stracture of amenable Banach algebras. J. London. Math. Soc. (2) 40, (1989), 89-104.

[R] M. R. Rieffel, Induced Banach representatios of Banach algebras and locally compact groups. J. Func. Analysis. 1(1967), 443-491.

Received: May 11, 2006 